almitrine caused increased periodic breathing. This might be expected if it augments peripheral chemoreceptor response. Acetazolamide decreases periodic breathing which is consistent with its action at the central chemoreceptors. Acetazolamide is a superior drug for elimination of periodic breathing and severe hypoxaemia during sleep at high altitude.
(The full version of this paper has been submitted toㄹㄹㄹ the American Review of Respiratory Disease fore publication)

\title{
Changes in body weight, fat and muscle mass at high altitude and the effect of acetazolamide (Abstract)
}

\author{
P.W. Dykes, N. Binns, A.R. Bradwell, J. Busby, P.J.G. Forster, J.J. Milles and N.V. \\ Richardson
}

The General Hospital, Steelhouse Lane, Birmingham B4 6NH, UK.

Body weight changes were studied during walking ascent and descent to $4846 \mathrm{~m}$ in 19 subjects, ten of whom were taking acetazolamide. Body fat was assessed using fat fold calipers at three skin locations. Muscle thickness was measured in both arms and legs using a portable linear array ultrasound unit. At high altitude weight was maintained in subjects on acetazolamide but tended to fall in subjects on placebo. During descent there was marked weight loss in both groups; mean loss $3.2 \mathrm{~kg}$ in subjects on acetazolamide and $4.1 \mathrm{~kg}$ in those on placebo. Body fat tended to fall but only by $0.12 \mathrm{~kg}$ and $0.4 \mathrm{~kg}$ in the two groups respectively. Muscle thickness in the thigh

\section{Reference}

Bradwell, A.R., Dykes, P.W., Coote, J.H. et al. Effect of acetazolamide on exercise performance and muscle mass at high altitude. Lancet 1986, i; 1001-1005. was significantly reduced at high altitude by $3.4 \mathrm{~mm}$ $(8.5 \%)$ in the acetazolamide group and $4.9 \mathrm{~mm}$ $(12.9 \%)$ in the placebo group. Loss of muscle in the upper arm was less, but the difference between groups was greater. This muscle wasting colitiv account for the greater part of the overall weight less At high altitude calorie intake was surprisingly lowa approximately $2000 \mathrm{~K}$ cal daily but with adequate protein $(73 \mathrm{~g} /$ day $)$. It remains to be shown whethen muscle loss can be prevented by dietary manipulation but a significant beneficial effect of acetazolamide has been demonstrated. 